\title{
Feasibility of Modified Remotely Monitored In-Home Gaming Technology for Improving Hand Function in Adolescents With Cerebral Palsy
}

\author{
Meghan Huber, Bryan Rabin, Student Member, IEEE, Ciprian Docan, Grigore C. Burdea, Senior Member, IEEE, \\ Moustafa AbdelBaky, Student Member, IEEE, and Meredith R. Golomb
}

\begin{abstract}
The convergence of game technology, the Internet, and rehabilitation science forms the second-generation virtual rehabilitation framework. This paper presents the first pilot study designed to look at the feasibility of at-home use of gaming technology adapted to address hand impairments in adolescents with hemiplegia due to perinatal stroke or intraventricular hemorrhage. Three participants trained at home for approximately $30 \mathrm{~min} / \mathrm{day}$, several days a week, for six to ten months. During therapy, they wore a Fifths Dimension Technologies Ultra sensing glove and played custom-developed Java 3D games on a modified PlayStation 3. The games were designed to accommodate the participants' limited range of motion, and to improve finger range and speed of motion. Trials took place in Indiana, while monitoFring/data storage took place at Rutgers Tele-Rehabilitation Institute (New Jersey). Significant improvements in finger range of motion (as measured by the sensing glove) were associated with self- and family-reported improvements in activities of daily living. In online subjective evaluations, participants indicated that they liked the system ease of use, clarity of instructions, and appropriate length of exercising. Other telerehabilitation studies are compared to this study and its technology challenges. Directions for future research are included.
\end{abstract}

Index Terms-Cerebral palsy, game console, hemiplegia, sensing glove, telerehabilitation.

\section{INTRODUCTION}

$\mathbf{C}$ HILDREN can develop hemiplegia from a perinatal brain injury or a later injury that affects only one side of their brain. When the injury causing hemiplegia occurs in the perinatal period or first few years of life, the resulting hemiplegia is known as "hemiplegic cerebral palsy." It is estimated that of the almost 800,000 children and adults living with cerebral palsy

Manuscript received May 29, 2009; revised September 19, 2009 and November 7, 2009. Current version published March 17, 2010. This work was supported in part by the National Institutes of Health under Grant K23 NS048024 and by the Clarian Foundation.

M. Huber was with the Department of Biomedical Engineering, Rutgers University, Piscataway, NJ 08854 USA (e-mail: mehuber@ caip.rutgers.edu).

B. Rabin was with the Department of Electrical and Computer Engineering, Rutgers University, Piscataway, NJ 08854 USA. He is now with the Department of Biomedical Engineering, Rutgers University, Piscataway, NJ 08854 USA (e-mail: brabin@caip.rutgers.edu).

C. Docan, G. C. Burdea, and M. AbdelBaky are with the Department of Electrical and Computer Engineering, Rutgers University, Piscataway, NJ 08854 USA (e-mail: docan@caip.rutgers.edu; burdea@caip.rutgers.edu; deesha@caip.rutgers.edu).

M. R. Golomb is with the School of Medicine, Indiana University, Indianapolis, IN 46202 USA (e-mail: mgolomb@iupui.edu).

This paper has a supplementary downloadable video available at http://ieeexplore.ieee.org, provided by the author. Contact burdea@caip.rutgers. edu for further questions about this work.

Digital Object Identifier 10.1109/TITB.2009.2038995 in the United States [1], approximately one-quarter are hemiplegic [2]. One disabling aspect of their disease is difficulty with activities of daily living (ADLs), most such tasks using both hands [3].

Constraint-induced movement therapy (CIMT) is one form of training, which has been shown to be effective for hemiplegic children and adults [4]-[6]. However, some CIMT trials did not include clients with severe hemiplegia, and would have excluded the severely hemiplegic adolescents who participated in the study described here [7], [8]. Treatment of the plegic limb with botulinum toxin injections [9] or surgery such as tendon transfer [10] may improve function, but are expensive. The effect of botulium toxin or surgery may be temporary without ongoing occupational and physical therapy [11]. What is needed is an affordable, accessible therapy that is well tolerated by children and adolescents, and can be done over the duration needed to obtain results. Furthermore, such an intervention needs to be inclusive of clients with very limited function of their plegic hand.

The ability to perform therapy at home is important in order to facilitate the training duration needed, without financial burden or without severely impacting the client's ability to participate in other activities. For adolescents with mild to severe hemiplegia and impaired hand function, how long is therapy beneficial and at what point a plateau is reached remain open questions.

Virtual reality use in physical therapy has been recognized as a way to provide a customized, highly motivating environment that also allows transparent real-time data collection and its storage in remote databases [12], [13]. Its clinical adoption has been slow so far due, in part, to the relatively high cost of current commercial systems used in therapy (\$10 000-\$50 000 [14], [15]). Although commercial technology is lagging, a number of "ad hoc" efforts are exploring game consoles-based systems as a lower cost alternative. These include the use of a modified Logitech haptic joystick [16], the PlayStation 2 EyeToy camera [17], and more recently, use of the Wii [18]-[20].

None of the aforementioned systems (including the Wii) specifically train finger movement. Furthermore, the use of offthe-shelf gaming technology in rehabilitation, though intuitively attractive, poses a number of problems. Off-the-shelf games are not designed for telerehabilitation purposes, thus they do not have the gradation and data collection capability a therapist may desire. More importantly, these games were designed for able, young (and generally fit) users. They may be too difficult and thus demoralizing for the client. Furthermore, the unsupervised 
use of game consoles may lead to overexertion, trauma, tendonitis (recently called "Wiiitis" [21]), high blood pressure, and other secondary problems [22].

As the technology is new, no long-term studies have been undertaken to investigate the effects of commercial game technology use by people with disabilities. This is of special concern to players with multiple medical problems. In the meantime, rehabilitation practice could use game consoles that operate under telerehabilitation conditions (to ensure monitoring and data collection at home), and are able to run games designed from the start for therapeutic use. Morrow and colleagues adapted an Xbox and a P5 glove [23] for hand rehabilitation [24]. Drawbacks of this earlier attempt were the limited computing power of the first-generation Xbox, an unreliable sensing glove, and the expense of hardware modifications needed to run the Linux operating system (OS). Furthermore, the Xbox-P5 setup was not used in telerehabilitation conditions.

The system presented here is a follow-up collaboration of Rutgers and Indiana Universities to develop a PlayStation 3based (PS3) system for hand telerehabilitation of children and adolescents with hemiplegia due to cerebral palsy. The major aims of this feasibility study were to: 1) examine acceptance of this gaming technology by adolescents with cerebral palsy and 2) examine potential changes in impairment and hand functioning following the use of this gaming technology. Though not a major aim of this study, the influence of longer durations of rehabilitation on participants' compliance with at-home therapy schedule data were collected.

\section{METHODS}

\section{A. Hardware Setup}

The PS3 telerehabilitation system developed for this study is shown in Fig. 1 [25]. Each of the participant's home had a PS3, a Fifth Dimension Technologies (5DT) 5 Ultra sensing glove [26], a flat panel TV, mouse, keyboard, and a digital subscriber line (DSL) modem used for connection to the Internet. The PS3 was chosen due to its ability to run Linux needed for customization and its multicore computation power useful in the real-time virtual reality simulations. At the same time, the number of PS3 systems already in the homes of potential users increased the chance of technology adoption if the therapy proved to have positive effects on clients with hemiplegic cerebral palsy.

The 5DT 5 Ultra glove was selected due to its lower cost compared to other commercially available sensing gloves, and the willingness of the manufacturer to build custom child-size gloves needed by two of the participants. The PS3 universal serial bus (USB) ports were used to plug in the keyboard, the mouse, a USB-to-serial adapter, and a subsequent serial-to-USB adapter for the glove. These adapters were needed since the glove had a USB hardware connection, but came with Linux drivers only for serial ports.

Three home telerehabilitation systems were installed in rural Indiana, while an identical system was placed at Indiana University (Indianapolis) for use as a backup. Another PS3-based rehabilitation system was used in game programming and testing at Rutgers Tele-Rehabilitation Institute. Data from the three

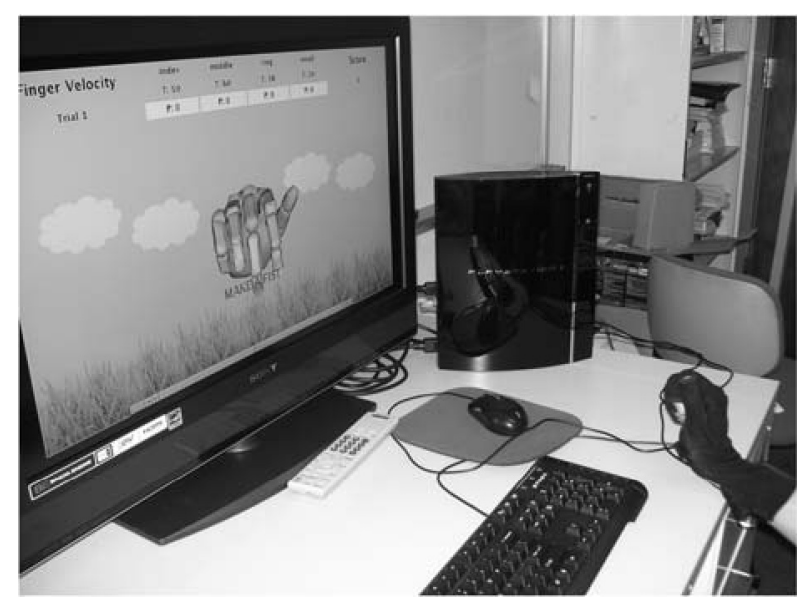

Fig. 1. Experimental home rehabilitation station [25]. Copyright Rutgers TeleRehabilitation Institute. Reprinted by permission.

homes were uploaded to a clinical database server at Rutgers. The clinical server was a Dell quad core workstation (model XPS 720) with redundant array of independent disks (RAID) hard drives, uninterruptible power supply and external one terabyte hard drive backup.

\section{B. Software Setup}

A number of software changes were needed to transform the PS3 game console into a rehabilitation station. These included changing the operating system to ensure openness, integrating drivers for the sensing glove, developing customized therapyoriented games, and implementing session baselines and a session scheduler. To allow telerehabilitation, additional softwares were written for data upload to the database server, as well as downloads of game upgrades and other software changes into the home PS3s.

1) PS3-Modified OS: Commercial gaming development for the PS3 uses the Sony Software Development Kit (SDK). This option was not taken here due to cost (\$10 000) and SDK inability to accommodate the sensing glove. Instead, the Yellow Dog Linux 6.0 (YDL) [27] was used, due to its optimization for the PS3 cell processors and relatively small memory footprint. The associated performance limitation is that the PS3 graphics processor is not accessed (Sony does not allow it). Thus, the custom rehabilitation games developed for the project were rendered in software by the PowerPC embedded on the multicore chip, and therefore had limited scene complexity.

At the application level, customization relates to the development of a serial communication protocol that would work with the glove USB port and Linux. To make the interface for the glove independent of the other devices already connected to the PS3, a daemon was customized to recognize the glove firmware and always assign to it the same device name ("glove").

2) Glove Calibration and Baseline Exercise: The 5DT 5 Ultra glove has one fiber-optic sensor per finger, which measures the "total" flexion or extension of each finger. This total flexion is due to a combination of the flexion of the finger 
metacarpal joint (MCP), the proximal interphalangeal joint (PIP), and the distal interphalangeal joint (DIP). Each sensor reading represents an integer from 0 to 4095 due to the analog-todigital conversion electronics embedded in the glove circuitry. Tests performed at Rutgers showed that the glove had high resolution, being able to detect changes in fingertip position as small as $0.12 \mathrm{~mm}$. However, the range of integers corresponding to a full flexion of the glove changed from finger to finger, due to the position of the sensing element within the glove fabric. Moreover, each glove had its own "signature," meaning that the same finger on two gloves would return different integer values for the same flexing amount. Although measurements showed that the integers fell somewhere between 600 and 4090 in all cases, it was necessary to determine the relationship between these integers and the finger flexing angle for each finger and each glove. Fig. 2 shows a sample of the integer-to-angle mapping for the index and thumb of a 5DT Ultra standard size glove. Both fingers show a linear relationship between raw integer data values and flexing angle values. This linear relationship was confirmed by bending the sensors across the acceptable range of angles in small intervals and obtaining the correlating raw data values. The sensitivity, however, is very different for each finger.

Apart from determining the relationship between glove readings and flexing angles, a glove calibration was needed before each training session commenced. This second calibration was done in order to map each rehabilitation game to the capabilities of each participant, in a particular day. While running the baseline exercise, the participant was given on-screen cues to flex/extend (open/close) his/her hand or bend/extend the thumb. The resulting calibration file mapped the participant's limited range of hand movement to the full range of movement of an on-screen hand avatar. The games used a percentage of the participant's flexion/extension range as threshold for completing each trial. These percentages, set empirically at the start of the study, could be modified to allow customization of the games and setting difficulty levels.

3) Session Scheduler: The session scheduler was displayed to the participant after running the baseline exercise. The scheduler allowed the participant to choose which game to play next (including difficulty level and number of trials) or to run the baseline exercise again if needed. This variability was chosen to empower the client and help reduce the boredom factor involved in repetitive rehabilitation applications over the many months of training. The total session time was displayed by the scheduler to show how long the participant had been training so far on that day. This helped the participants determined if they met the recommended length of therapy per day.

4) Training Games: Custom games were written in Java3D [28], which required the PowerPC Java version provided by IBM and an open source implementation of the Java3D specifications (version 1.3). Several Java 3D exercises were developed based on experience with games authored at Rutgers in earlier studies for adult poststroke clients in the chronic phase [29], [30].

Three games were tested as follows.

Finger range of motion game: The finger range of motion game [see Fig. 3(a)] [25] asked participants to start by making
Thumb
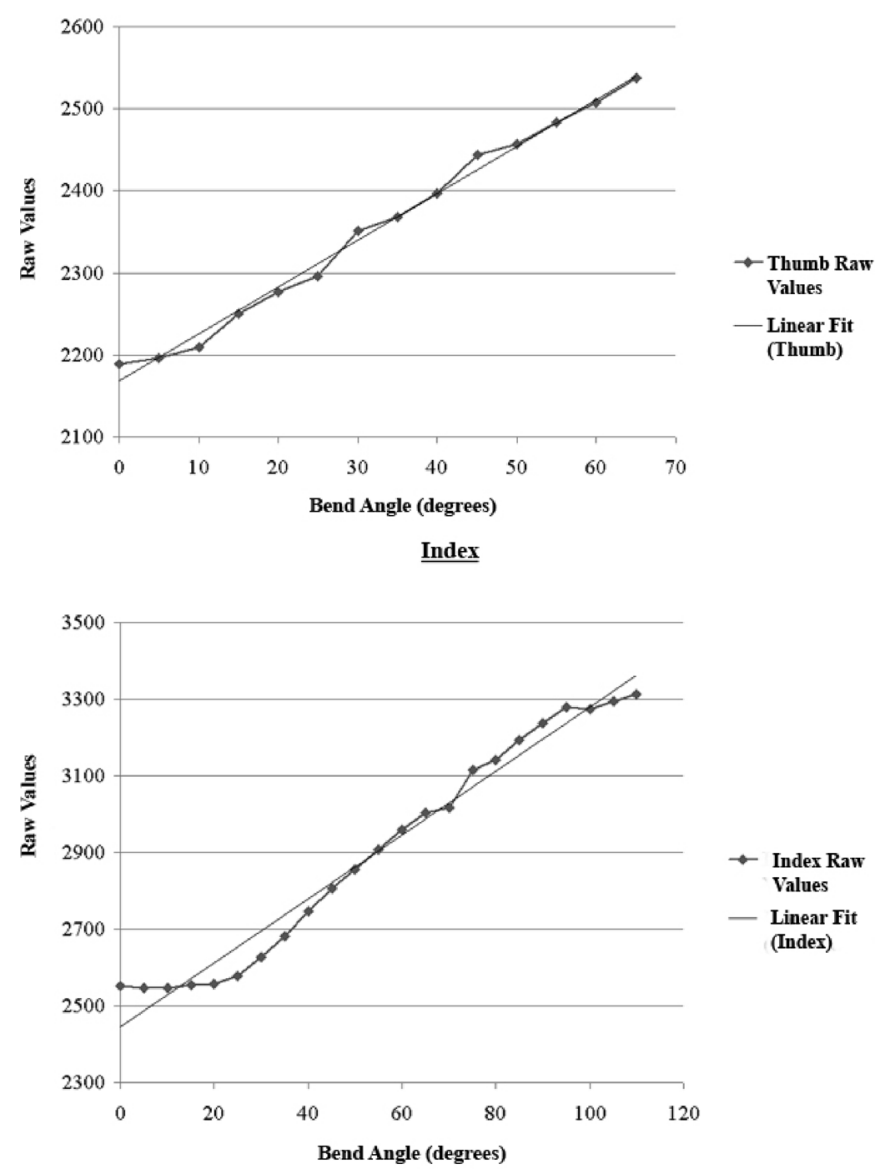

Fig. 2. 5DT glove does not measure joint angles directly, rather it returns integers. The relationship between raw sensor readings and finger "total" flexing angle is linear and finger dependent. (Top) Index; (bottom) thumb. Copyright Rutgers Tele-Rehabilitation Institute. Reprinted by permission.

a fist (when extension was trained) or to first open their hand as much as possible (when flexion was trained). Subsequently, each of the four fingers was mapped to a bar of "dirty" pixels, which occluded a portion of a pleasant image. Images were randomized to maintain interest and chosen from scenes familiar to the participants (corn, Indiana 500 racetrack, Indianapolis landscape, favorite pets, etc.). The task was to "clean up" the screen, uncovering the image in proportion to each finger contribution. This provided an easily understood knowledge of results (KR). When the exercise trained the thumb, the whole image was uncovered proportional to the amount of thumb movement. Additional KR was provided numerically by the graphical user interface (GUI) at the top portion of the screen. This GUI displayed numerically finger-specific range data, comparing current finger results to target values. The GUI also displayed the total exercise time for that game. A "window cleaning" sound was played to add realism and increase immersion into the game.

Finger velocity training games: The "butterfly game" [see Fig. 3(b)] asked the participant to first make a fist (if extension was trained) or to open their hand as much as possible (if flexion was trained). Subsequently, virtual butterflies appeared from the 


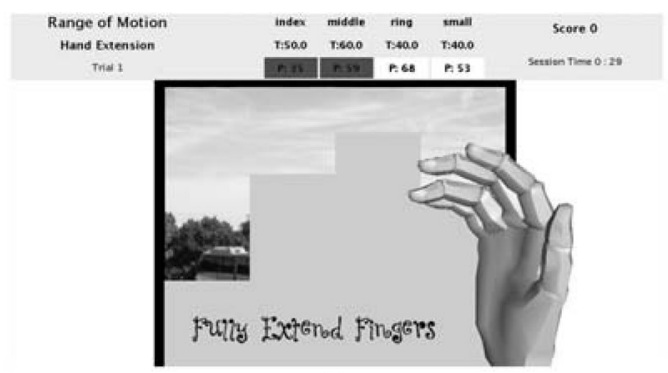

(a)

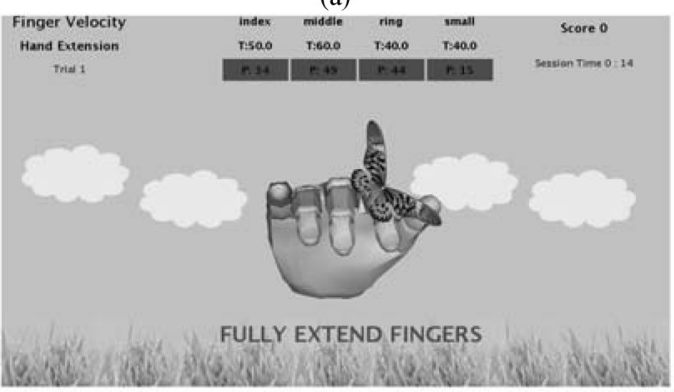

(b)

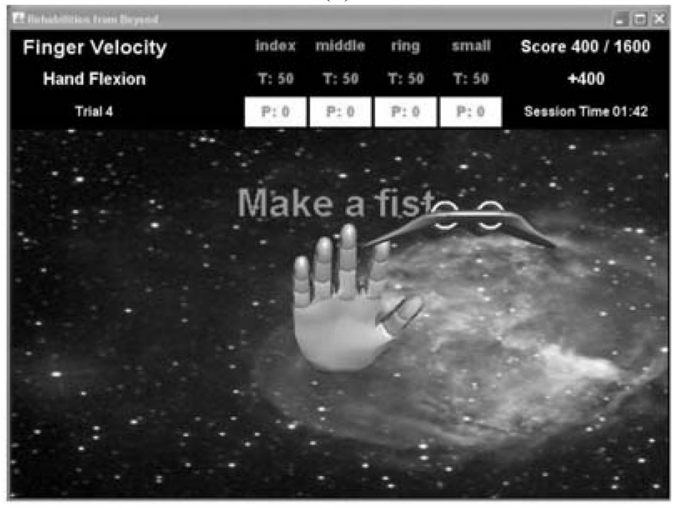

(c)

Fig. 3. Virtual reality finger exercises: (a) range of motion (sliders); (b) finger velocity (butterfly); and (c) finger velocity (UFO) [25]. Copyright Rutgers TeleRehabilitation Institute. Reprinted by permission.

side of the screen and needed to be "scared away" by moving the fingers or the thumb fast. As long as the participant achieved the set flexion/extension goals before the butterfly reached the hand avatar, it would fly away. Otherwise, the butterfly flew back to the hand and had to be scared away again. After a number of butterflies had been scared away, a mosquito attempted to sting the hand avatar. The participant had to move the fingers fast enough to scare the mosquito away, else the hand flashed red and a corresponding unpleasant sound was produced. The difficulty of the game was increased by making the butterflies or the mosquitoes fly faster, thus requiring faster finger movement due to shorter reaction time. The GUI was similar to that of the previous game, and congratulatory text was displayed at exercise end.

A second version of the velocity training game used unidentified flying objects (UFOs) instead of butterflies [Fig. 3(c)]. In the "bonus" round, the UFO beamed a "shrink ray" if the participant had not opened/closed the hand fast enough. The hand avatar then turned green and shrank. If, however, the participant had moved the fingers fast, the UFO flew away and crashed (with a corresponding explosion sound). The participants preferred this version of the game as the scene was more appealing to them.

5) Remote Clinical Database and Remote Monitoring: The clinical database stored computerized data generated from the games and the periodic online subjective evaluation surveys. The database module consisted of:

1) a Java program on the PS3s, which uploaded the baseline exercise and game data every night;

2) a Java program on the clinical server, which received these data;

3) another Java program, which parsed these files, extracted, and stored the data on the local database;

4) a Java program on the clinical server, which made sure that participants played according to schedule; and

5) an online subjective evaluation questionnaire, which ran on the PS3 twice a month.

The visualization of clinical data was done remotely using an integrated Web portal running on the clinical server. This was a secure Web-based application, wherefrom an authorized user could view participant data and graph relevant variables. These graphs showed game-specific configurations (such as difficulty level) and client progress over time.

With servers running on the local and background displays, the remote monitoring feature of the system could be used to either watch what was happening on the participant's screen in real time or make changes for software updates. This functionality was used extensively in the project, as exercise software was continually upgraded, or bugs fixed.

A method for notification by e-mail and/or SMS text message alerted the clinicians when a client failed to $\log$ in for a set number of days. This insured redundancy and consistency with the database method that notified the clinician if the participants did not keep up their regular home rehabilitation schedule.

6) Overall System Cost: Excluding the remote database server and Internet service provider costs, a PS3-based hand rehabilitation system is estimated to cost \$2500 (\$400 PS3, \$1000 5DT glove, $\$ 200$ adaptors for the glove, $\$ 400$ high-definition TV (HDTV), \$500 software). These are, of course, prototype costs, as the system is not commercially available at the time of this writing.

\section{Recruitment}

The study received approval from the Institutional Review Boards at Indiana University and Rutgers University. Subsequently, three adolescents (two boys, one girl), age 13-15, with moderate-severe hemiplegic cerebral palsy were recruited by their pediatric neurologists (including author Golomb) at Riley Hospital for Children. All three had cerebral palsy due to perinatal brain injury and became symptomatic during their first year of life. Two (participants 1 and 3 ) had perinatal stroke, and participant 2 had been a premature infant with intraventricular hemorrhage. All three had difficulty opening and closing their plegic hand, and struggled to pick up objects with that hand. The three participants were chosen because they had good cognitive function and were highly motivated to participate in the study; 
TABLE I

Game Play Duration and Finger Range Changes by Participant Over

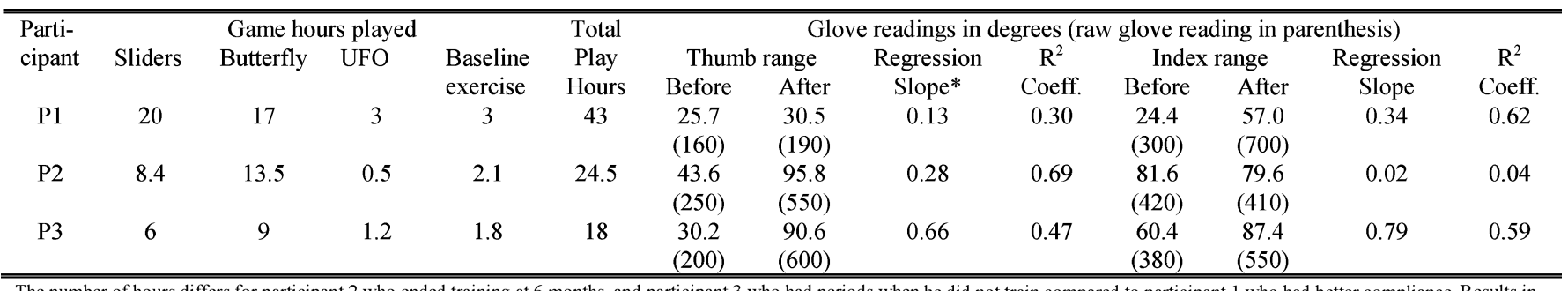

The number of hours differs for participant 2 who ended training at 6 months, and participant 3 who had periods when he did not train compared to participant 1 who had better compliance. Results in this table refer to thumb and index, judged by the authors as most clinically relevant. Angles are "global" rather than joint-based, since the sensing glove used has one sensor for each finger. The glove provides raw integers which are then converted to degrees based on glove calibration. The $\mathrm{R}^{2}$ coefficient is a statistical measurement used to determine how well the linear progression model fits the actual data

*A positive regression slope signifies finger range improvement over the months of therapy.

all three were good students in age-appropriate school classes, and only one qualified for extra help for mild learning issues. Their medical background has been described in greater detail previously [31]. All participants were told to practice for approximately 30 min daily (including rest time), five days per week. They, together with their caregivers, signed consent/assent forms and completed a familiarization session at Indiana University. Subsequently, the research team traveled to their homes, installed, and tested the hand rehabilitation systems.

\section{OUTCOMES}

Detailed descriptions of outcomes after the first three months of therapy were described elsewhere [31]. Outcomes reported here are for six to ten months of home telerehabilitation and refer to the following.

1) Changes in finger range of motion as measured by the glove: Finger range of motion was inferred from the raw numbers (integers) returned by the sensing glove during the baseline exercise at the start of each session. Range of motion could not be measured with a goniometer due to the subjects' extreme spasticity. The children could not assume the testing position to measure range of motion and utilized compensatory movements, which made getting an accurate measurement very difficult.

2) Changes in ADL performed with the affected hand: The ADL data were collected by asking the participants and their families if they were able to use the affected hand for a selected number of tasks, before the start of telerehabilitation training, then at three months of training, and at ten months into the project. No standardized forms were used to either collect or analyze the data besides the answers the researchers received. The tasks chosen were based on the clinical judgment of the physician coauthor (Golomb), who sees people with cerebral palsy and other motor disabilities in the clinic. Tasks important to activities of daily living of adolescents, which involve the use of the upper extremity (UE), were put on the list.

3) Subjective evaluation of the system: Every two weeks, the participants were prompted by an online subjective evaluation questionnaire which they had to fill before continuing their session. There were nine questions, scored 1 (least desirable outcome) to 7 (most desirable outcome).
4) Occupational therapist (OT) assessments: A pediatric OT examined the three participants prior to the start of telerehabilitation, and then at three months into the study; these results were reported in detail elsewhere [31]. These included the Jebsen test of hand function [32] and grasp strength measurements using a Jamar dynamometer. Participant 1 , who had practiced longest, was also formally evaluated by the OT at ten months of training.

Statistical analysis: The statistical analyses were generated using a custom MATLAB program (Table I and Fig. 4). This program uses its built-in statistical functions to perform a leastsquares polynomial fit to the bending angle data obtained from the baseline exercise over the course of the first six months of training. A linear fit is shown to illustrate the overall rate of change during this time period. A linear regression with a positive slope in this interval correlates to an increase in finger range of movement. The $R^{2}$ correlation coefficient is measured to determine how well this linear model fits the actual data.

\section{RESULTS}

\section{A. Aim I: Adoption of Gaming Technology by the Participants}

1) Practice Time and Game Usage: Participants 1 and 3 remained enrolled for ten months, and participant 2 remained enrolled for six months. Participant 1 logged a total of $43 \mathrm{~h}$ (baseline exercise and play, but excluding the rest periods), participant 2 logged $24.5 \mathrm{~h}$ over six months, while participant 3 totaled $18 \mathrm{~h}$ over ten months. During this time, the games were played differently by each, a result of game and system availability and client's preference. The games were introduced at different times, with "Sliders" first (March 23, 2008), "Butterfly" shortly after (April 1, 2008), and UFO later (July 20, 2008). Table I shows the distribution of game hours logged by each participant. The Sliders game was designed to train range of movement with no time constraint. Conversely, the Butterfly and UFO games had a time constraint for reaching a threshold, training speed of movement. Taking in consideration that not all games were introduced at the same time, Butterfly was the most played game by the participants, while both Sliders and Butterfly were effective in training range and speed of movement.

2) Subjective Evaluation of the Telerehabilitation System: The subjective evaluation responses varied with participant and month of study. Table II gives the average score and range of 
responses for each question. The average score for the system overall for all participants was 5.6 out of 7 .

3) Issues Limiting the Use of Game Technology: Maintaining participants' interest was key to keep them motivated; games were modified and new images introduced on the Sliders game to motivate subjects. Social issues were an important factor limiting game time. All three subjects were busy with homework and school activities. Participant 2 withdrew after six months because she moved to an older home with wiring problems, which could not support the technology. Participant 3 was dealing with an illness in the family. These issues affected compliance [33]. In a perfect compliance scenario, each subject should have logged $11 \mathrm{~h} /$ month, totaling $111 \mathrm{~h}$ for participants 1 and 3 , and $66 \mathrm{~h}$ for participant 2.

\section{B. Aim II: Changes in Hand Impairment and Function}

1) Changes in Remotely Measured Finger Range of Movement: Of the large amount of data, Fig. 4 shows only range changes for the thumb and index (fingers most relevant clinically). These were measured for the first six months of training for all three participants during session baseline exercises. The graphs show the difference in degrees as measured by the glove for a participant's maximum flexion and maximum extension (the larger the difference, the larger the range of finger movement between flexion and extension). The slope of the linear regression line fit to the raw data range over time, as seen in Fig. 4 and quantified in Table I, provides a measure of increased ability. A slope near zero corresponds to no measurable increase in ability. An increase in the participant's ability will yield an increase in the positive slope of this angle range over time. The low $R^{2}$ values in Table I point to the need to utilize a model of higher degree since there are a number of variables that determine the participant's progress and ability from day to day. This purely linear model, along with the technical issues found with prolonged use of the sensing gloves, yield a low correlation coefficient. This is due to large variability from the linear model, but does give some insight into the participant's overall progress during the extended period of study. Future studies will help to refine this model to allow for a more accurate representation of the participant's progress over the duration of the study. The measurements in Table I do correlate with the increased ability seen in OT assessment, as well as increased ability in ADL activity. After six months, the glove measurements degraded (due to wear and tear, displaced sensors, or broken connectors). While gloves were repaired or replaced, the confidence in glove data after six months is low; thus, these data are not included here.

2) Changes in ADL: Table III shows the participants' ability to perform ADLs with their affected hand as reported by subjects and their families.

3) OT Assessments: Formal OT measurements for grip, pincer strength and the Jebsen test of hand function were performed at prestudy baseline and at three months, and have been reported previously [31]. During the first three months, hand grasp strength measure with a dynamometer improved in all three participants, and all three showed improvements on
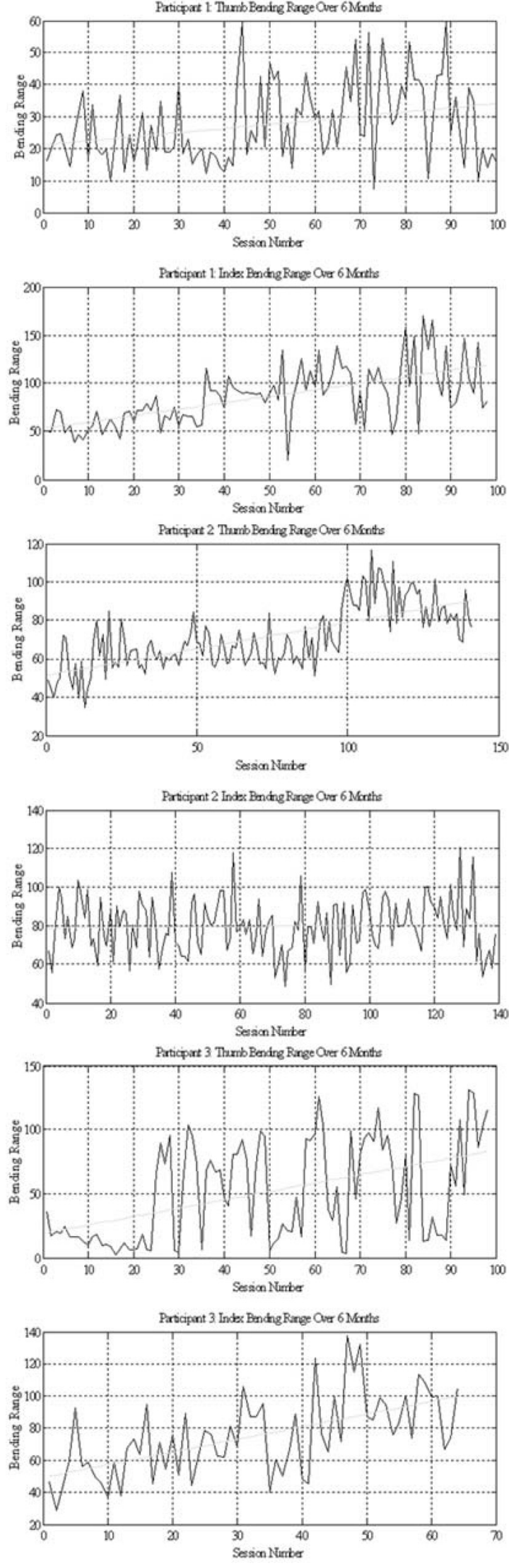

Fig. 4. Changes in thumb and index range of motion for the three participants over the six months of home telerehabilitation. Copyright Rutgers TeleRehabilitation Institute. Reprinted by permission. 
TABLE II

OnLine SubJective Evaluation SCORES COMPLETEd By the PARTICIPANTS OVER SiX MONTHS OF HOME TELEREHABILITATION

\begin{tabular}{|c|c|c|c|c|c|}
\hline \multirow[t]{2}{*}{ No } & \multirow[t]{2}{*}{ Question } & & 2 & 3 & \multirow[b]{2}{*}{$\begin{array}{l}\text { Avg. } \\
\text { Score } \\
\text { All } 3\end{array}$} \\
\hline & & \multicolumn{3}{|c|}{$\begin{array}{l}\text { Participant's } \\
\text { Avg. Score } \\
\text { and range }\end{array}$} & \\
\hline \multirow[t]{2}{*}{1} & Was the system easy to use? & 6.5 & 6.4 & 6.2 & 6.4 \\
\hline & (1-estremely difficult; 7 -extremely easy) & $4-7$ & $5-7$ & $3-7$ & \\
\hline \multirow[t]{2}{*}{2} & Did you find the games interesting? & 5.9 & 5.4 & 6.2 & 5.2 \\
\hline & (1-not at all; 7-extremely interesting) & $5-7$ & $4-6$ & $3-7$ & \\
\hline \multirow[t]{2}{*}{3} & Did you have muscle pain and & 6.2 & 6.2 & 5.4 & 5.9 \\
\hline & discomfort? (1-frequent pain; 7-no pain) & $4-7$ & $4-7$ & $4-6$ & \\
\hline \multirow[t]{3}{*}{4} & Were the instructions given to you & 6.9 & 7 & 6.1 & 6.7 \\
\hline & useful? & 6-7 & 7 & $4-7$ & \\
\hline & (1-not at all useful; 7-extremely useful) & & & & \\
\hline \multirow[t]{2}{*}{5} & Were you bored while exercising? & 4.7 & 4.2 & 4.3 & 4.4 \\
\hline & (1-extremely bored; 7-not at all bored) & $1-7$ & $2-6$ & $3-6$ & \\
\hline \multirow[t]{2}{*}{6} & Was the length of exercising in a day & 6.4 & 6.6 & 6.4 & 6.4 \\
\hline & $\begin{array}{l}\text { appropriate (1-not at all appropriate; } \\
7 \text {-extremely appropriate) }\end{array}$ & $5-7$ & $6-7$ & $5-7$ & \\
\hline \multirow[t]{2}{*}{7} & Were there many technical problems? & 2.4 & 3 & 3.1 & 2.8 \\
\hline & (1- Frequent problems; 7-no problems & $1-4$ & $2-5$ & $2-5$ & \\
\hline \multirow[t]{2}{*}{8} & Would you encourage another patient to & 6.7 & 7 & 4.2 & 6 \\
\hline & $\begin{array}{l}\text { use it? (1-not at all; 7-strongly } \\
\text { encourage) }\end{array}$ & 6-7 & 7 & $3-5$ & \\
\hline \multirow[t]{2}{*}{9} & Do you think this is a good system? & 6.6 & 6.6 & 5.6 & 6.3 \\
\hline & (1-very poor; 7-excellent system) & $5-7$ & $6-7$ & $5-7$ & \\
\hline
\end{tabular}

For each participant and question, top bold number is the average of responses score and bottom number signifies the range of response scores ( 1 is the lease desirable outcome and 7 the most desirable outcome). The last column represents the average of scores for a given question for al participants.

TABLE III

PARTICIPANTS' ADL CARRIED OUt With the IMPAIRED HAND

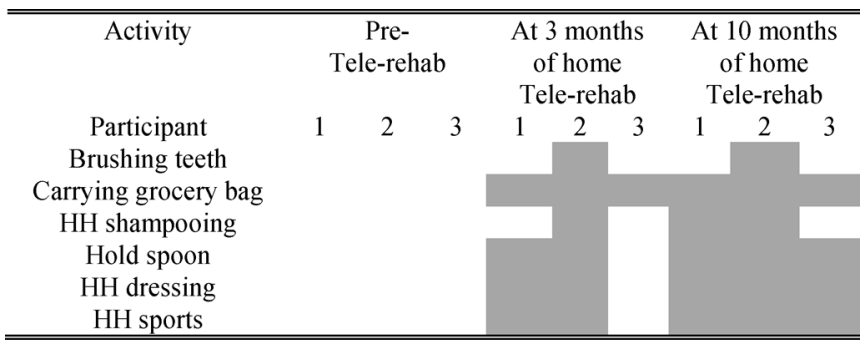

Shaded areas represent activities participants were able to perform, either with the impaired hand alone or as a helper hand (hh) to aid the unimpaired hand. For each participant, this self-report refers to abilities before the telerehabilitation intervention, after three months of training and again after ten months (in part adapted from [25])

multiple tasks of the Jebsen test. Two of the participants progressed from being unable to lift large, heavy objects (a task on the Jebsen test) to being able to do so. Participant 1 formal evaluation at ten months of training showed his ability to lift large, heavy objects continued to improve. He was also able to lift bags and open doors (see attached video).

\section{DisCUSSION AND Future DiRECTIONS}

This pilot study describes six to ten months of home hand telerehabilitation in three clients with cerebral palsy. Results suggest that this form of telerehabilitation is feasible, accept- able to clients, and may improve hand function. Although technical difficulties were encountered, the participants still rated the system highly.

To the authors' knowledge, studies of such length of time are rare. For example, Durfee [34] used telerehabilitation at home to improve finger function in adults poststroke. Patients used finger and wrist joint motions to control the cursor in a tracking task. Participants trained for several hours each day over the course of ten days. While the training was intensive, it did not involve a game console or virtual reality games. Holden used virtual reality in a telerehabilitation of the UE of chronic patients poststroke [35]. Participants trained using magnetic trackers on their UE and instrumented objects, and imitated expert examples presented on a display. No specific finger data were obtained. In Holden's study, participants trained for a total of $30 \mathrm{~h}$ over six weeks. By comparison, in this CP telerehabilitation study, participant 1 trained more hours spread over ten months. Participant 2 interrupted training after six months due to a move to a new home, and the third participant trained over ten months, but with interruptions due to significant family issues, which made him intermittently stop training. Remote computer monitoring showed this and helped researchers motivate the client to resume training. The role telerehabilitation plays in increasing the participant's compliance has been recognized by the industry [36]. Winters [37] believes that compliance with telerehabilitation regimes is a complex process. It is influenced by the participant's home environment and disruptions thereof, by the complexity of the intervention, as well as by the ratio of patient's perceived gains versus cost and effort associated with remote rehabilitation. The present feasibility study provides further evidence of the complex nature of telerehabilitation compliance and the important role played by the home environment and technology used.

In this study, the technology differed from the home telerehabilitation studies cited before. In this case, additional complicating factors were technical problems with the glove sensors, which would pull out of their guides providing unreliable readings. Durfee and Holden's studies did not use gloves, and thus did not have to deal with this technology-limitation issue. Furthermore, since they did not use game consoles, they did not encounter issues with limited available memory or closed graphics resources.

Despite the aforementioned difficulties, months of playing therapeutic games on the PS3 at home produced improved hand function for all of the participants with hemiplegic cerebral palsy. This is exemplified by the increase in range of motion as sensed by the 5DT glove (see Fig. 4) and sustained by the occupational therapy evaluation (Jebsen subset). There is a wide variability in participants 1 and 3 range of motion measures over time, which may have been caused by a combination of the way the gloves were donned, whether glove sensors were damaged, or by interruption in daily practice (due to causes explained earlier). Some of the difficulty with the glove data was due to the indirect way of inferring finger angles. These were not measured directly, but determined from manual calibration used to convert raw integer readings (this may have added computation artifacts). 
Although detailed clinical data were presented elsewhere [31], it is worth noting that two participants who were unable to lift large heavy objects before game-based telerehabilitation could do so posttraining. Participant 1 continued to improve, and at ten months could lift large heavy objects faster. His mother reported he was participating more in household chores and using both hands to do them. The improvement in hand function, which is assumed to be primarily due to significant increase in finger range of motion, resulted in improvements in the participants' ability to perform ADLs. While Table III is based on nonstandardized ADL forms, it nonetheless shows increased capacity to perform tasks, as gauged by: 1) client and care giver reports and 2) observation during taping of task execution. The shaded area shows activities that participants could do at three months, but were unable to do at the start of the study. At ten months, the participants were again asked the same ADL questions. Participant 2 maintained her gains despite the fact that she had stopped therapy four months prior. This is indicative of retention. Participant 3, who had interrupted training but later resumed it, was now able to hold a spoon and use his affected hand as helper hand for dressing and sports, something he could not do before. Participant 1, who had trained continuously for ten months, was now able to also use his hand as helper hand when shampooing. The improvement of participant 1 hand function following the ten months of training is apparent in the video accompanying this article.

With the length of therapy and the technical difficulties encountered, it is interesting to look at the subjective evaluation of the system (Table II). Participants 1 and 2 were substantially less bored at the beginning (6) than at six months (4). Participant 3 did not have data at six months, but was constantly more bored (score 4 at start and 4 at ten months). Getting more bored was to be expected as game choice was limited. However, when asked, "Would you encourage other patients to use it?" they answered 6, with 7 being "strongly encourage." This rating was maintained at 6 and 7 over the six months for participants 1 and 2. Participant 3 started at 5, went lower (3), but ended at 5 at ten months. Their overall impression of the system was 6.3 (7 being "excellent system"). This is remarkable considering that the participants were adolescents who had only to choose between three therapeutic games, and had practiced over a long duration. This apparent paradox may be due to the lack of tailored therapies for this client population (as in no home therapy at all), so participants felt this experimental system was very useful to them. Further work is needed to determine if gained improvements are maintained one year later.

This was a feasibility study, rather than a controlled one, so no control subjects were included in the study. The question one can contemplate is whether the results obtained through the VR game therapy are any different from if (theoretically) the control subjects had practiced opening and closing their hand for a similar duration? While it is hard to speculate, it can be hypothesized that it is unlikely that adolescents would have dedicated the same length of time practicing repetitive movements in the absence of a related visual-compelling stimulus or in the absence of remote supervision.
Results of this study support further investigation as follows.

1) Finding a glove more suitable to the abnormal hand configuration of clients with Cerebral Palsy. Such a glove needs to be easier to put on and take off, and be more rugged than the $5 \mathrm{DT}$ glove used in the present study.

2) Developing new rehabilitation games, including games to train finger fractionation (independent finger movement), endurance, power output (the ability to exert mechanical work over time), and force exertion. With more game choice, the clients will also have more freedom as far as what they choose to play, and a broader range of training methods will be explored.

3) Finding a more open computing configuration than the PS3. Either Sony Co., the game console manufacturer, will allow access to the graphics hardware or platforms that provide sufficient RAM and easy system programming will be investigated.

\section{ACKNOWLEDGMENT}

The authors would like to thank M. E. Nwosu and M. BarkatMasih for communicating with the participants and their families to determine what issues had developed over time.

\section{REFERENCES}

[1] United Cerebral Palsy. Cerebral palsy fact sheet. (2007). [Online]. Available: http://www.ucp.org/uploads/cp_fact_sheet.pdf

[2] M. Yeargin-Allsopp, K. Van Naarden Braun, N. S. Doernberg, R. E. Benedict, R. S. Kirby, and M. S. Durkin, "Prevalence of cerebral palsy in 8year-old children in three areas of the United States in 2002: A multisite collaboration," Pediatrics, vol. 121, pp. 547-554, 2008.

[3] B. R. Van Zelst, M. D. Miller, R. N. Russo, S. Murchland, and M. Crotty, "Activities of daily living in children with hemiplegic cerebral palsy: A cross-sectional evaluation using the assessment of motor and process skills," Develop. Med. Child Neurol., vol. 48, no. 9, pp. 723-727, 2006.

[4] B. Bonnier, A. C. Eliasson, and L. Krumlinde-Sundholm, "Effects of constraint-induced movement therapy in adolescents with hemiplegic cerebral palsy: A day camp model," Scan. J. Occup. Therapy, vol. 13 no. 1, pp. 13-22, 2006.

[5] S. C. Deluca, K. Echols, C. R. Law, and S. L. Ramey, "Intensive pediatric constraint-induced therapy for children with cerebral palsy: randomized, controlled, crossover trial," J. Child Neurol., vol. 21, no. 11, pp. 931-938, 2006.

[6] E. Taub, S. L. Ramey, S. DeLuca, and K. Echols, "Efficacy of constraintinduced movement therapy for children with cerebral palsy with asymmetric motor impairment," Pediatrics, vol. 113, no. 2, pp. 305-312, 2004

[7] S. L. Wolf, C. J. Winstein, J. P. Miller, E. Taub, G. Uswatte, D. Morris, C. Giuliani, K. E. Light, and D. Nichols-Larsen, "Effect of constraintinduced movement therapy on upper extremity function 3 to 9 month after stroke: The EXCITE randomized clinical trial," J. Amer. Med. Assoc. vol. 296, pp. 2095-2104, 2006.

[8] A. M. Gordon, J. Charles, and S. L. Wolf, "Efficacy of constraint-based movement therapy on involved upper-extremity use in children with hemiplegic cerebral palsy is not age-dependent," Pediatrics, vol. 117, no. 3e, pp. 363-373, Mar. 2006.

[9] M. A. Wallen, S. J. O'Flaherty, and M. C. Waugh, "Functional outcomes of intramuscular botulinum toxin type A in the upper limbs of children with cerebral palsy: A phase II trial," Arch. Phys. Med. Rehabil., vol. 85, no. 2, pp. 192-200, 2004.

[10] E. A. Zancolli, "Surgical management of the hand in infantile spastic hemiplegia," Hand Clin., vol. 19, no. 4, pp. 609-629, 2003.

[11] T. Y. Chin, J. A. Duncan, B. R. Johnstone, and H. K. Graham, "Management of the upper limb in cerebral palsy," J. Pediatr. Orthop. B, vol. 14, no. 6, pp. 389-340, 2005.

[12] G. Burdea, "Keynote address: Virtual rehabilitation: Benefits and challenges," J. Methods Inf. Med., vol. 42, pp. 519-523, 2003. 
[13] M. Holden, "Virtual environments for motor rehabilitation: Review," $C y$ berPsychol. Behav., vol. 8, no. 3, pp. 187-211, 2005.

[14] Gesturetek Coo. (2006). IREX Introduction [Online]. Available: http://www.gesturetek.com/irex/introduction.php

[15] Hocoma AG. (2008). Armeo ${ }^{\circledR}$ —Functional upper extremity rehabilitation [Online]. Available: http://www.hocoma.ch

[16] D. Reinkensmeyer, C. T. Pang, J. Nessler, and C. C. Painter, "Web-based telerehabilitation for the upper extremity after stroke," IEEE Trans. Neural Syst. Rehabil. Eng., vol. 10, no. 2, pp. 102-108, Jun. 2002.

[17] D. Rand, R. Kizony, and P. L. Weiss, "Virtual reality rehabilitation for all: Vivid GX versus Sony PlaStation II EyeToy," in Proc. 5th Int. Conf. Disabil., Virtual Real. Assoc. Tech., U.K., 2004, pp. 87-94.

[18] J. Sinnema, "Wii technology used in brain rehabilitation," Edmonton J. (2007, May). [Online]. Available: http://www.canada.com/ edmontonjournal/news/story.html?id=5ff7f35b-e86b-4264-b3e6-19f6b5 $075928 \& \mathrm{k}=63173$

[19] L. Zyga. (2007). The healing power of Wii-Interactive gaming is helping injured vets on the road to recovery. PC Mag. [Online]. Available: http://www.pcmag.com/article2/028172170784,00.asp

[20] J. E. Deutsch, M. Borbely, J. Filler, K. Huhn, and P. Guarrera-Bowlby, "Use of a low-cost, commercially available gaming console (Wii) for rehabilitation of an adolescent with cerebral palsy," Phys. Therapy, vol. 88, no. 10, pp. 1196-1207, 2008.

[21] J. Bonis, "Acute Wiiitis," N. Eng. J. Med, vol. 356, pp. 2431-2432, 2007.

[22] Nintendo Wii-Health and Safety Precautions. (2010). [Online]. Available: http://www.nintendo.com/consumer/wiisafety.jsp

[23] The Robot Group. (2005). P5 Glove [Online]. Available: http://www. robotgroup.net/index.cgi/P5Glove

[24] K. Morrow, C. Docan, G. Burdea, and A. Merians, "Low-cost virtual rehabilitation of the hand for patients post-stroke," in Proc. 5th Int. Workshop Virtual Rehabil., New York, 2006, pp. 6-10.

[25] M. Huber, B. Rabin, C. Docan, G. Burdea, M. Nwosu, M. Abdelbaky, and M. Golomb, "PlayStation 3-based telerehabilitation for children with hemiplegia," in Proc. Virtual Rehab., 2008, pp. 105-112.

[26] 5DT Co. (2007). 5DT data glove ultra series [Online]. Available: http://www.5dt.com/downloads/

[27] Fixstars. Yellow Dog Linux (2010). [Online]. Available: http://www. fixstars.com/en/products/ydl/

[28] J. Barrilleaux, 3D User Interfaces With Java 3D. Greenwich, CT: Manning, 2000.

[29] D. Jack, R. Boian, A. Merians, M. Tremaine, G. Burdea, S. Adamovich, M. Recce, and H. Poizner, "Virtual reality-enhanced stroke rehabilitation," IEEE Trans. Neural Syst. Rehabil. Eng., vol. 9, no. 3, pp. 308-318, Sep. 2001.

[30] A. Merians, D. Jack, R. Boian, M. Tremaine, G. Burdea, S. Adamovich, M. Recce, and H. Poizner, "Virtual reality-Augmented rehabilitation for patients following stroke," Phys. Therapy, vol. 82, no. 9, pp. 898-915, 2002.

[31] M. R. Golomb, B. C. McDonald, S. J. Stuart, J. Warden, J. Yonkman, A. Saykin, B. Shirley, M. Huber, B. Rabin, M. AbdelBaky, M. Nwosu, M. Barkat-Masih, and G. Burdea, "In-home virtual reality videogame telerehabilitation in children with hemiplegic cerebral palsy," Arch. Phys. Med. Rehabil., vol. 91, pp. 1-18, Jan. 2010.

[32] I. E. Asher, "Jebsen hand function test," in Occupational Therapy Assessment Tools: An Annotated Index, 2nd ed. Bethesda, MD: Amer. Occup. Therapy Assoc., p. 154, 1996.

[33] M. R. Golomb, M. Barkat-Masih, B. Rabin, M. Abdelbaky, M. Huber, and G. Burdea, "Eleven months of home virtual telerehabilitation-Lessons learned," in Proc. Virtual Rehabil. Int. Conf., Haifa, Israel, pp. 23-28, 2009.

[34] W. Durfee, S. A. Weinstein, J. R. Carey, E. Bhatt, and A. Nagpal, "Home stroke telerehabilitation system to train recovery of hand function," in Proc IEEE 9th Int. Conf. Rehabil. Robot., pp. 353-356, 2005.

[35] M. Holden, T. A. Dyar, and L. Dayan-Cimadoro, "Telerehabilitation using a virtual environment improves upper extremity function in patients with stroke," IEEE Trans. Neural Syst. Rehabil. Eng., vol. 15, no. 1, pp. 36-42, Mar. 2007.

[36] K. Debevois. (2006, Oct. 17). Telerehab: Therapy anywhere, anytime adding a new dimension to patient compliance with telerehabilitation. Therapy Times [Online]. Available: http://www.therapytimes.com/content $=6501 \mathrm{~J} 64 \mathrm{E} 487 \mathrm{EA} 4941$

[37] J. Winters. (2009). Models of telehealth encounters, or tele-encounters Marquette Univ., Milwaukee, WI [Online]. Available: http://www. eng.mu.edu/rehab/Rehab167/Mod3/model/

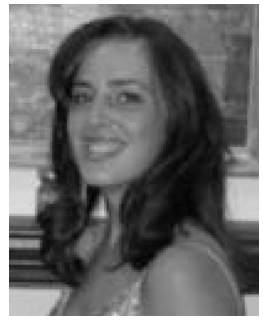

Meghan Huber received the B.S. degree in biomedical engineering from Rutgers University, Piscataway, NJ.

She was with the Human-Machine Interface Laboratory, Rutgers University, as a Research Assistant, where she was a Teaching Assistant for the virtual reality course in the Electrical and Computer Engineering Department.

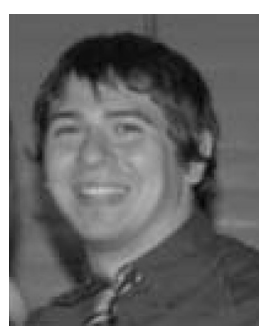

Bryan Rabin (S'09) received the B.S. degree in electrical and computer engineering in 2007 from Rutgers University, Piscataway, NJ, where he is currently working toward a second major in the field of biomedical engineering.

He was student volunteer at the 2008 Virtual Rehabilitation International Conference, Vancouver, BC, Canada.

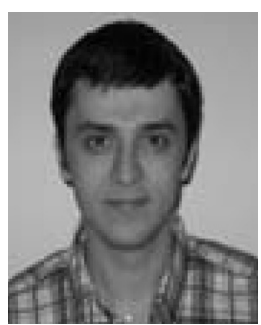

Ciprian Docan received the B.S. degree from Bucharest Polytechnic University, Bucharest, Romania, in 2005, and the M.S. degree in electrical and computer engineering in 2008 from Rutgers University, Piscataway, NJ, where he is currently working toward the Ph.D. degree in the Electrical and Computer Engineering Department.

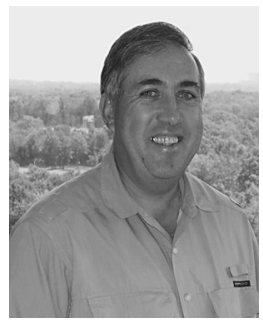

Grigore C. Burdea (M'86-SM'89) received the $\mathrm{Ph} . \mathrm{D}$. degree in applied science from New York University, New York City, in 1987.

$\mathrm{He}$ is currently an Electrical and Computer Engineering Professor at Rutgers University, Piscataway, NJ, where he founded the Human-Machine Interface Laboratory, which produced novel haptic interfaces for the arm, hand, and ankle, and later Rutgers TeleRehabilitation Institute.

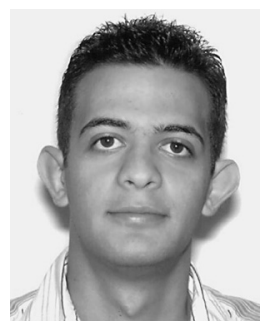

Moustafa AbdelBaky (S'05) received the B.S. degrees in electrical and computer engineering, and computer science in 2008 from Rutgers University, Piscataway, NJ, where he is currently working toward the graduate degree.

$\mathrm{He}$ is Graduate Assistant in the Electrical and Computer Engineering Department, Rutgers University.

Meredith R. Golomb received the M.D. degree from Harvard Medical School, Boston, MA, in 1994, and the M.Sc. degree in clinical epidemiology from the University of Toronto, Toronto, ON, Canada.

She was a trainee in pediatrics at the Children's Hospital Los Angeles, and in adult and child neurology at Massachusetts General Hospital. She was a Pediatric Stroke Fellow at The Hospital for Sick Children, Toronto. She is currently an Associate Professor of pediatric neurology in the School of Medicine, Indiana University, Indianapolis. 